\title{
Capacity Evaluation and Optimization of Heavy Haul Railway Station Based on the Simulation of Operation of Station
}

\author{
Mengqi $\mathrm{Ma}^{2}$ Xingchen Zhang, Zhimei Wang \\ School of Traffic and Transportation, Beijing Jiaotong University, Beijing 100044, China \\ a15120855@bjtu.edu.cn
}

Keywords: Rely on the fixed and flexible application principles of track.

\begin{abstract}
Heavy haul railway mainly transport bulk cargo such as coal and steel. Its characteristics includes large volume of freight, complicated operation of station, obvious heavy cargo direction etc. The bulk cargo's timeliness standard is low, the occupied time between different operations of station is distinct different. Therefore, the heavy haul railway train has great optimization space in the transportation organization of the station. The purpose of this paper is to strengthen the capacity of heavy haul railway stations. Based on the analysis of operations of the heavy load railway station, the simulation system is established. Rely on the fixed and flexible application principles of track, the shortest path selection method of route and the train insertion method to strengthen the train station capacity. The simulation is carried out with CiYaowan station in Baoshen Railway as an example, the feasibility of the method and simulation system is verified.
\end{abstract}

\section{Introduction}

For the research of the capacity of the station, previous studies have focused on the study of high speed railway passenger stations. Passenger station have accurate arrival time and departure time of train, with clear time constraints and fixed track use constraints, the operations is not so completed[1] [4]. Most of the research on freight stations through the analytical calculation method, the related research involved in the operation of the station is limited to the simple train arrival and shunting operation[5] [7]. Therefore, this paper is aimed at the simulation of the complex station operation of the heavy haul railway station, including angle folding train operation and ten thousand ton train combination operation, basis on which, the capacity of the station is evaluated and optimized.

\section{Analysis of Operations}

\subsection{The Basic Types of Operation and Process}

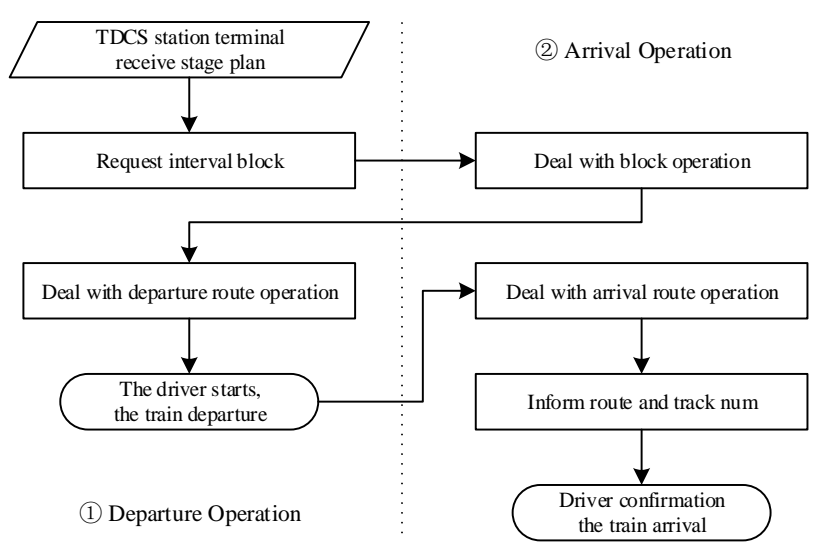

Figure 1. Process of arrival and departure operation

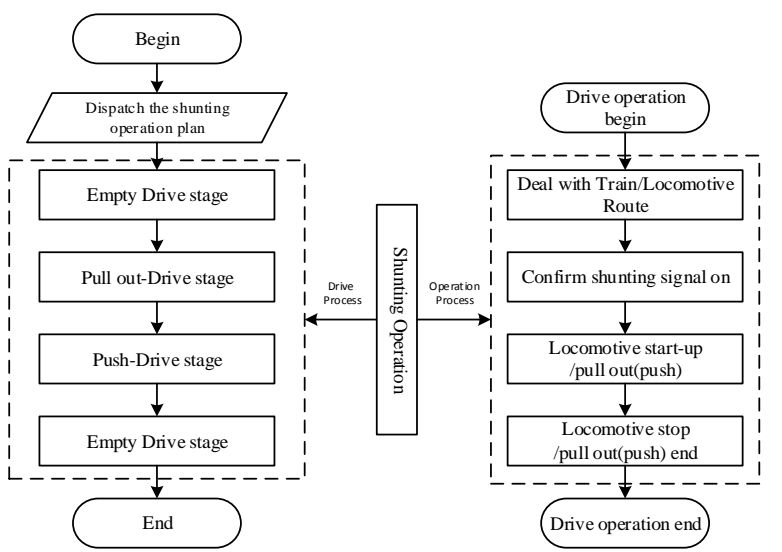

Figure 2. Shunting operation process

The basic operation types of heavy haul railway section stations generally include arrival and departure operation (Figure 1), shunting operation (Figure 2), check operation, tail operation locomotive operation and so on. 


\subsection{Angle Folding Train Operation and Ten Thousand Ton Train Combination Operation}

Angle folding train operation and train combination operation include basic types of operation, as shown in TABLE1.

Table 1. Complex operation flow chart of station

\begin{tabular}{|c|c|}
\hline Type & Technological process \\
\hline $\begin{array}{l}\text { Angle folding train } \\
\text { operation }\end{array}$ & (1)-(4)-(5)-(6) 1 -(3)-(6) ${ }^{2}-(4)-(5)^{1}-(2)$ \\
\hline Combined train operation & $\begin{array}{l}\text { Front train:(1)-(4)-(5)-(7)²-Whole Train, Latter Train:(1)-(4)-(5)-(3)-(7)33-(6)1-Whole } \\
\text { Train } \\
\text { Whole Train:(4)-(5)-(2) }\end{array}$ \\
\hline
\end{tabular}

(1)arrival operation(2)departure operation(3)shunting operation(4)check operation(5)tail operation (6)1locomotive removal operation(6)2locomotive hanger operation(7)1combined locomotive removal operation(7)2combined locomotive hanger operation(7)3 train hanger operation

\section{Simulation Model Establishment}

\subsection{Main Program Process}

As shown in Figure 3, input parameters to the system, mainly including simulation, station, train information in three aspects. Simulation information includes simulation step and simulation length of time; Station information includes initial status tables of equipment, equipment selection tables (TABLE 2, TABLE 3), parallel route table; Train information for initial train event table, including all of the train flow path, train type, planned time of arrival.

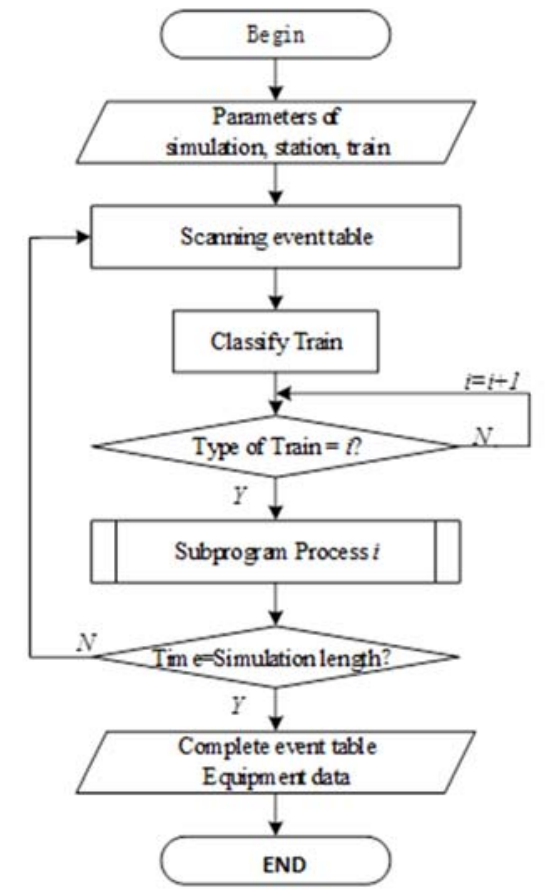

Figure 3. Main program flow chart

The simulation strategy adopts the process interaction method, with clock pushing the loop. Select and classify the train events in the present simulation step, identify the types of train events and operate according to their type subprograms, until all train events are processed in this step, proceed with clock pushing. After the end of the simulation time, the complete train event table is output, including the actual arrival and departure time, the selected equipment and the status records of equipment. Finally, based on the statistical calculation of the related train and station equipment in the simulation results, a new station capacity strengthening scheme is obtained based on the train insertion method.

\subsection{Subprogram Process}

The process of passing train 
Step1: In accordance with the track selection table and each track occupation status to determine whether a track can be occupied. If there is no, the train delay, decelerates or stops at the outside of the station, this train operation ends, the next train operation is carried out; if so, the next step is carried out.

Step2: In accordance with the route selection table and the occupancy status of the various routes and their conflicting route, it is possible to judge whether there is a way to take up. If there is no, then Step3 is carried out; if so, Step4 is carried out.

Step3: Judge whether the events are tried by all the available track. if so, the train delay, decelerates or stops at the outside of the station, this train operation ends, the next train operation; if not, the selected the track has been marked as not available, return to Step1.

Step4: According to the chosen track, route selection table, occupancy status of the various routes and their conflicting route, determine whether there is an available route. If not, change the property of the train to stopping train, update station equipment occupancy, change the property of the train to stopped train, make the stop time to be simulation step; if there is, update station equipment occupancy, this train operation ends.

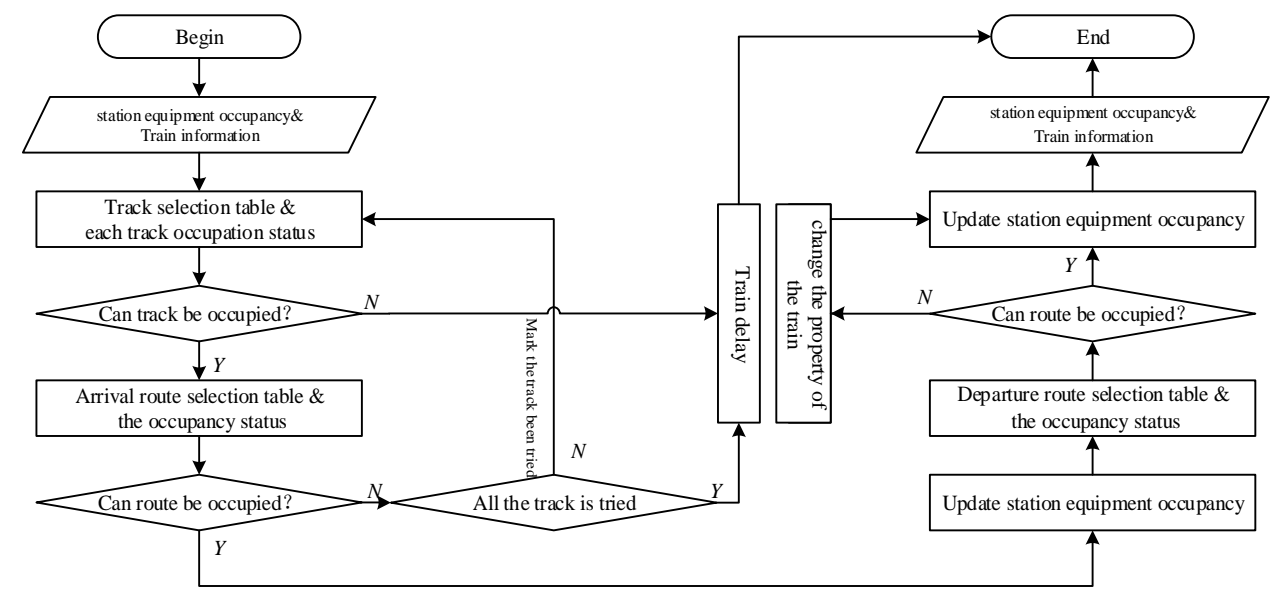

Figure 4. The process chart of passing train

The process of stopping train

Step1 Step3 is the same as the passing train process.

Step4: According to the chosen track and arrival route, calculate the planned departure time according to the stop time and update station equipment occupancy. After the operation of this train, the property of the train will change to stopped train automatically.

The process of stopped train

Step: According to the chosen track, route selection table, occupancy status of the various routes and their conflicting route, determine whether there is an available departure route.If not, the train departure delays, continue to stop in their tracks, the train track update its attribute, this train operation ends, the next train operation begins; if there is, update station equipment occupancy, this train departure operation ends. This step is equivalent to the last step of other types of trains.

The process of unit train

Step1 Step3 is the same as the passing train process.

Step4: Based on the selected route and stopped track, calculate the stopped time, update station equipment occupancy. According to the property of the train, it is judged whether the train of matching unit has stopped at the station. If so, trigger the locomotive departure event (locomotive depot / locomotive waiting track), carry on the next step; if not, the next step will be carried out directly.

Step5: Judge whether the train front unit train and latter unit train have arrived at the station. If not, then update station equipment occupancy, lock the track; if so, and change the property of the latter unit train to in-station train, the operation of unit train ends.

The process of in-station train 
Step1: Determine whether the route required for the in-station train in the station is available. If not, then push and traction operation delay; if so, then push and traction operation begin, update station equipment occupancy, next step is carried out.

Step2: Finish the related combination operation, update station equipment occupancy, and generate a new ten thousand ton train (change the property of the train to stopping train).

The process of arrival locomotive and departure locomotive

Step: In accordance with the route selection table and the occupancy status of the various routes and their conflicting route, it is possible to judge whether there is a route to take up. If there is no, the locomotive delay; If so, it updates the properties of the route.

The process of angle folding train and ten thousand ton combination train

It is composed of the above basic operations, as shown in Fig 5 and Fig 6

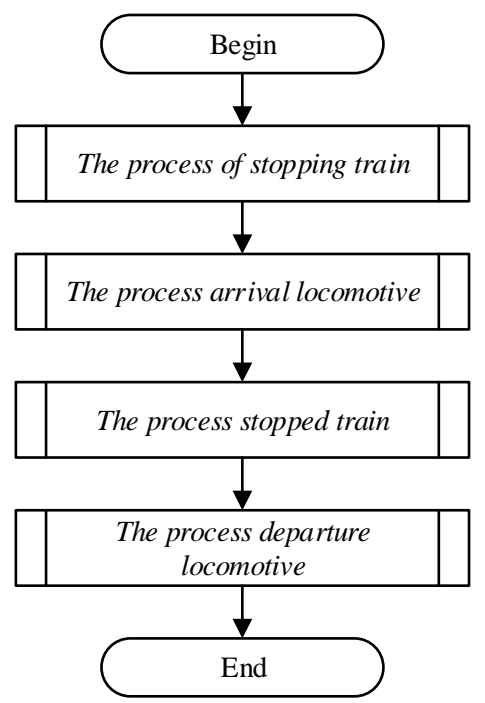

Figure 5. The process chart of passing train

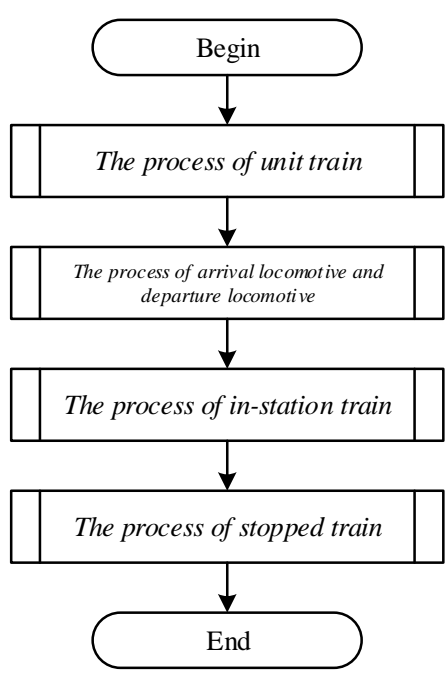

Figure 6. The process chart of passing train

\section{Optimization Method of Capacity}

\subsection{Track and Route Optimization}

Track selection rely on fixed and flexible use principles. Each train path is corresponding to a few frequently-used tracks. If all the frequently-used tracks are used, it is necessary to choose the other track without influence to other trains. The track selection table is shown as TABLE 2.

Table 2. The track selection table

\begin{tabular}{|c|c|c|}
\hline Train path & The track priority of passing train & The track priority of stopping train \\
\hline$[$ From,To $]$ & {$\left[L_{1}^{\text {固 }}, L_{2}^{\text {固 }}, \ldots, L_{m}^{\text {固 }} \quad L_{1}^{\text {灵 }}, L_{2}^{\text {灵 }}, \ldots L_{n}^{\text {灵 }}\right]$} & {$\left[L_{1}^{\text {固 }}, L_{2}^{\text {固 }}, \ldots, L_{m^{\prime}}^{\text {固 }} L_{1}^{\text {光 }}, L_{2}^{\text {灵 }}, \ldots L_{n^{\prime}}^{\text {尝 }}\right]$} \\
\hline
\end{tabular}

Route selection rely on the shortest path. The track is determined, the train is determined from section to track through the throat area. It becomes a shortest path problem that starting from the identified point to a determine point through the network, which can be solved by Floyd algorithm, giving the sorting sequence of the available routes. The route selection table is shown as TABLE 3

Table 3. The track selection table

\begin{tabular}{ccc}
\hline Train path & Track num & The route priority \\
\hline From / To & Track & {$\left[R_{1}, R_{2}, \ldots R_{p}\right]$} \\
\hline
\end{tabular}

\subsection{Track and Route Optimization}

Take the following 5 constraints to considering.

The operation time 


$$
\begin{array}{cc}
\left.\left\{L_{f} \mid L_{f^{\prime}}>\min \left(T_{l}\right)\right)\right\} & f=1,2, \ldots K, f^{\prime}=1,2, \ldots K^{\prime}, l=1,2 \\
N_{k}^{l}=\operatorname{int}\left(\frac{L_{f}}{T_{l}}\right) & f=1,2, \ldots K, l=1,2 \\
\operatorname{Tr}_{i}^{i n}=T_{i}\left(1-R_{i}\right) & i=1,2, \ldots N_{k}^{l}
\end{array}
$$

$f^{\prime}, f$ Represent the number of all and available time sequence numbers of the track, respectively. $l$ represents the type of the inserted train. $L_{f}$ represents every spare time of the track, $K^{\prime}$ in all. $L_{f}$ represents every spare time of the track, which can be used, $K$ in all. $T_{l}$ represents the operation time of the train $l$. $N_{k}^{l}$ represents the number of train $l$ can be inserted in the available time of the kth segment. $R_{i}$ represents the status of the various routes needed to be used, 0 represents it can be used, or it can't be used. $T r_{i}=1$ represents the planned inserted train can be inserted, or it can't be insert.

Running time in section

The up train and down train can't run at the same time in single track railways.

$$
\begin{aligned}
& T_{\text {arrive }}>T_{\text {aready_go }}+2 T_{\text {section }} \\
& T_{\text {go }}<T_{\text {aready_arive }}-2 T_{\text {section }}
\end{aligned}
$$

$T_{\text {arrive }}, T_{g o}$ represent the arrival time and departure time of the inserted train. $T_{\text {aready } g o}$ represents the arrival time and departure time of the already existing train which is earlier than the arrival time of the inserted train. $T_{\text {aready_arive }}$ represents the arrival time and departure time of the already existing train which is latter than the arrival time of the inserted train. $T_{\text {section }}$ represents the running time in section.

The interval of Train continuous departure time and arrival time

$$
T_{i}^{1}+I<T_{i}<T_{i}^{2}+I \quad i \in\{\text { arrive go }\}
$$

$T_{i}^{1}$ represents the departure(arrival) time of the already existing train which is earlier than the departure(arrival) time of the inserted train. $T_{i}^{2}$ represents the departure(arrival) time of the already existing train which is latter than the departure(arrival) time of the inserted train.

Maintenance time

$$
T_{i}<W_{\text {start }} \quad \text { or } \quad T_{i}>W_{\text {end }} \quad i \in\{\text { arrive go }\}
$$

$T_{i}$ represents the arrival time and departure time of the inserted train, $W_{\text {start }}, W_{\text {end }}$ represent the beginning and end of the maintenance time.

According to the track available time sequence in the simulation time, take the constraints to considering, rely on the track fixed and flexible use principles, insert the new trains. The related information include the type of the train $l$, arrival time $L_{f}^{\text {begin }}+\Delta t+(i-1) T_{l}$, and the train path[From,To], $L_{f}^{\text {begin }}$ represents the beginning time of the fth available time, $\Delta t$ represents the Operation time interval between front and latter two trains.

\section{Simulation Case Analysis}

According to TDCS data from September 2016 to September 2017, the CiYaowan Station was the longest-occupied station for the stock road, and July 6, 2017 was the longest day of the CiYaowan station in the whole year. So this paper takes the July 6, 2017 CiYaowan Station as an example to analyze the case. As shown in Figure 7, CiYaowan Station includes 7 tracks, left throat including 3 switch groups, 12 routes, Link Shigetai Station direction, the right throat including 5 switch groups, 22 routes, connect Wulanmulun Station, Shendong Station, the Halagou Dedicated Line three direction. The day CiYaowan through the train 111, divided into 5 kinds of traffic path: Shigetai to Wulanmulun 16, Shigetai to Shendong 27, Halagou to the Shendong 13, Wulanmulun to Shigetai 44, Wulanmulun to Halagou 11. 


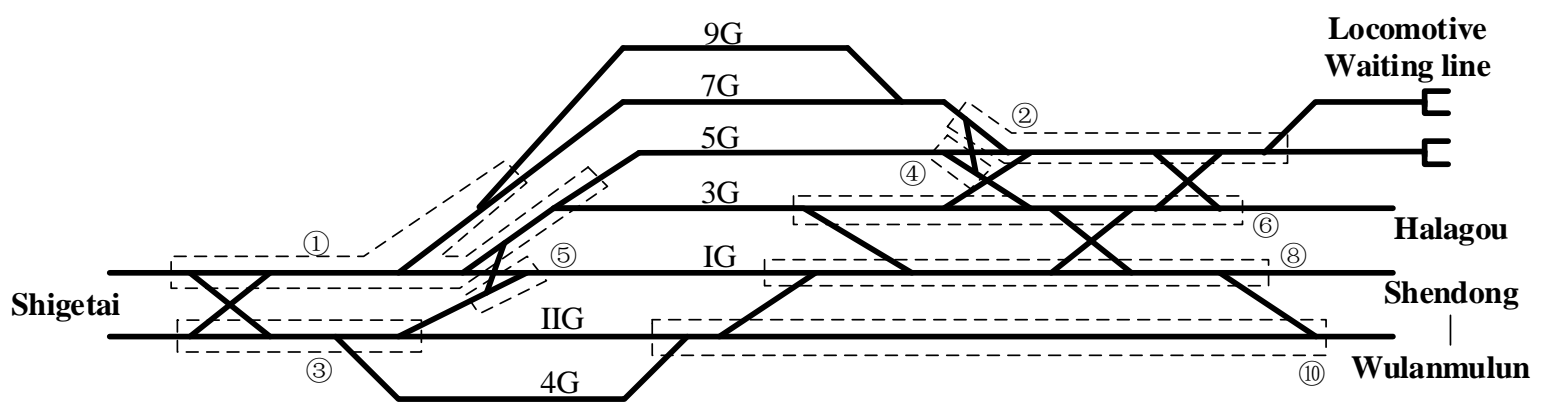

Figure 7. The diagram of Ciyaowan Station

The simulation time is $00: 00$ to $24: 00$, the maintenance time is $05: 00$ to $08: 20$. The train input scheme of 13 in TABLE 4 was designed for the station capability evaluation and optimization, and the relevant statistic indexes were obtained.

Table 4. The track selection table

\begin{tabular}{ccccccccccc}
\hline scheme & angle folding & combination & ordinary trains & unit train & combined train & $\begin{array}{c}\text { earlied } \\
\text { time/train }\end{array}$ & $\begin{array}{c}\text { Track } \\
\text { occupied }\end{array}$ & $\begin{array}{c}\text { utilization } \\
\text { of line }\end{array}$ & $\begin{array}{c}\text { switch } \\
\text { occupied } \\
\text { times }\end{array}$ & $\begin{array}{c}\text { var of } \\
\text { switch } \\
\text { occupied } \\
\text { times }\end{array}$ \\
\hline 0 & $20-200$ & $70-200$ & 111 & 0 & 0 & 0 & - & - & - & - \\
1 & 0 & 0 & 109 & 2 & 1 & 41 & 289 & $20.6 \%$ & 67 & 47.96 \\
2 & 20 & 70 & 109 & 2 & 1 & 37 & 407 & $32.0 \%$ & 67 & 49.47 \\
3 & 50 & 110 & 109 & 2 & 1 & 31 & 438 & $34.9 \%$ & 67 & 49.19 \\
4 & 100 & 150 & 109 & 2 & 1 & 23 & 558 & $46.7 \%$ & 66 & 49.44 \\
5 & 170 & 200 & 109 & 2 & 1 & 10 & 724 & $62.7 \%$ & 65 & 49.87 \\
6 & 200 & 200 & 109 & 2 & 1 & 5 & 827 & $72.7 \%$ & 66 & 49.66 \\
7 & 250 & 200 & 109 & 2 & 1 & -3 & 907 & $80.3 \%$ & 64 & 50.08 \\
8 & 50 & 110 & 164 & 2 & 1 & - & 584 & $47.5 \%$ & 105 & 82 \\
9 & 50 & 110 & 188 & 2 & 1 & - & 975 & $84.9 \%$ & 73 & 55.36 \\
10 & 50 & 110 & 107 & 4 & 2 & 29 & 456 & $36.8 \%$ & 67 & 49.56 \\
11 & 50 & 110 & 105 & 6 & 3 & 29 & 482 & $39.5 \%$ & 69 & 49.35 \\
12 & 50 & 110 & 103 & 8 & 4 & 29 & 507 & $42.1 \%$ & 69 & 48.57 \\
13 & 50 & 110 & 101 & 10 & 5 & 29 & 541 & $45.3 \%$ & 70 & 48.93 \\
\hline
\end{tabular}

Scheme 0 extracting data from TDCS, does not involve the time occupation information of the track and switch, so the average advance departure time of all trains is taken as the standard of the train route and the track selection optimization after the simulation optimization. And the design of the scheme 1 , the angle and the combination operation time is set to 0 , as a reference for judging the track and switch occupancy of other schemes.

From the scheme 1 to 7 , it can be seen that the increase in train operations time has led to a less time for the train to start early, increasing the utilization rate of the track, but the average occupancy of switch from scheme 4 began to decrease, indicating that there have been some trains delayed. The simulation results of scheme 7 show that every train has been delayed on average. Therefore, in order to further evaluate the ability and optimize the simulation, the time of Train angle folding operation and the combination operation are $50 \mathrm{~min}$ and $110 \mathrm{~min}$ respectively to ensure that the average operation time is not higher than the actual average operating time.

Schemes 8 and 9, respectively, are inserted into 55 and 33 trains on the basis of scheme 3 and 8 , respectively, by means of a train insertion method in the spare time section of the equipment. (i)Scheme 8 compared with scheme 3 (Fig 8, Fig 9), the average occupancy time of the track, the utilization rate of the hair line, the mean occupancy times of switch have been improved, and the standard deviation of switch is changed from 50 to 82, and the balance of switch utilization is reduced, The main reason for the increase in the number of trains through the train led to left throat 3rd switch group and the right end of the throat of the 10th switch group used too many times. (ii) Scheme 9 compared to 8, the number of switch occupied is less due to the track and route selection of passing train is preferred to other trains, passing train are too many leading to the route being delayed, some 
of the train cannot get out of the station, leading to the track has been occupied, cannot accept the other trains to the track.

So the station train's passing capacity is greater than 166 columns (127 passing train +37 angle folding train +2 unit train +1 combination train) less than 188 columns (147 passing train +39 angle fold train +2 columns to the train +1 combination train).

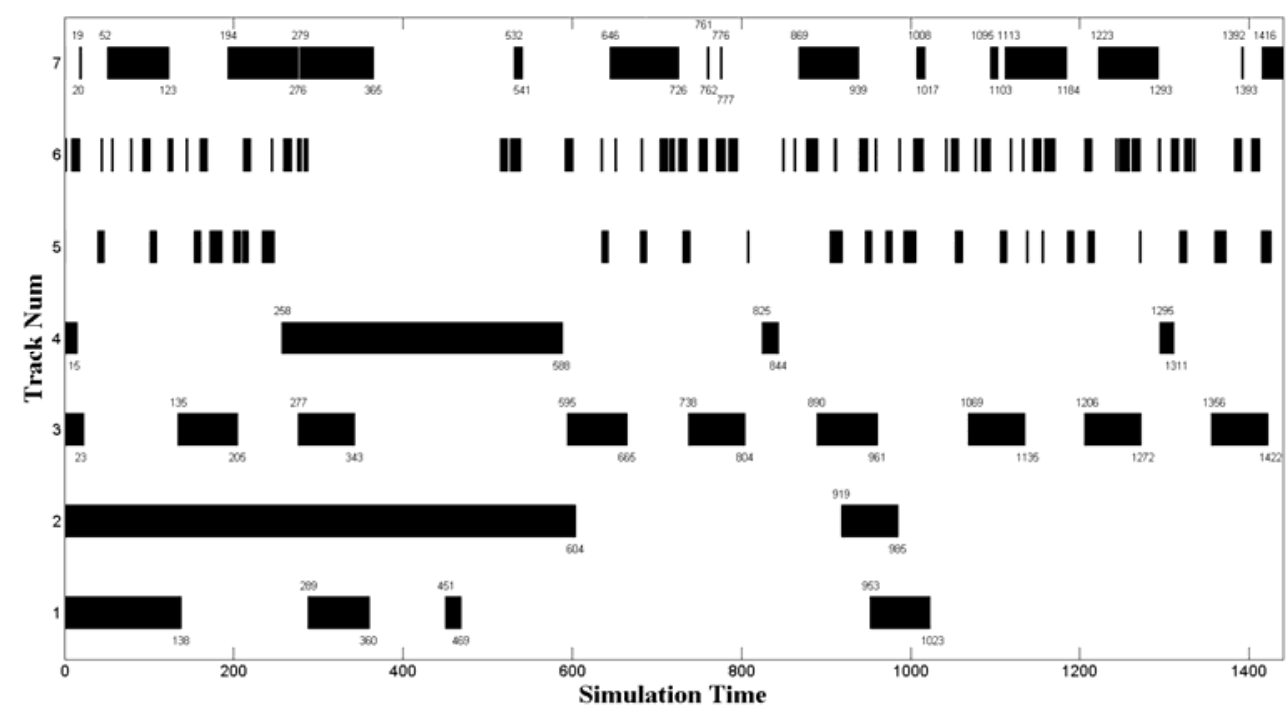

Figure 8. Scheme 3

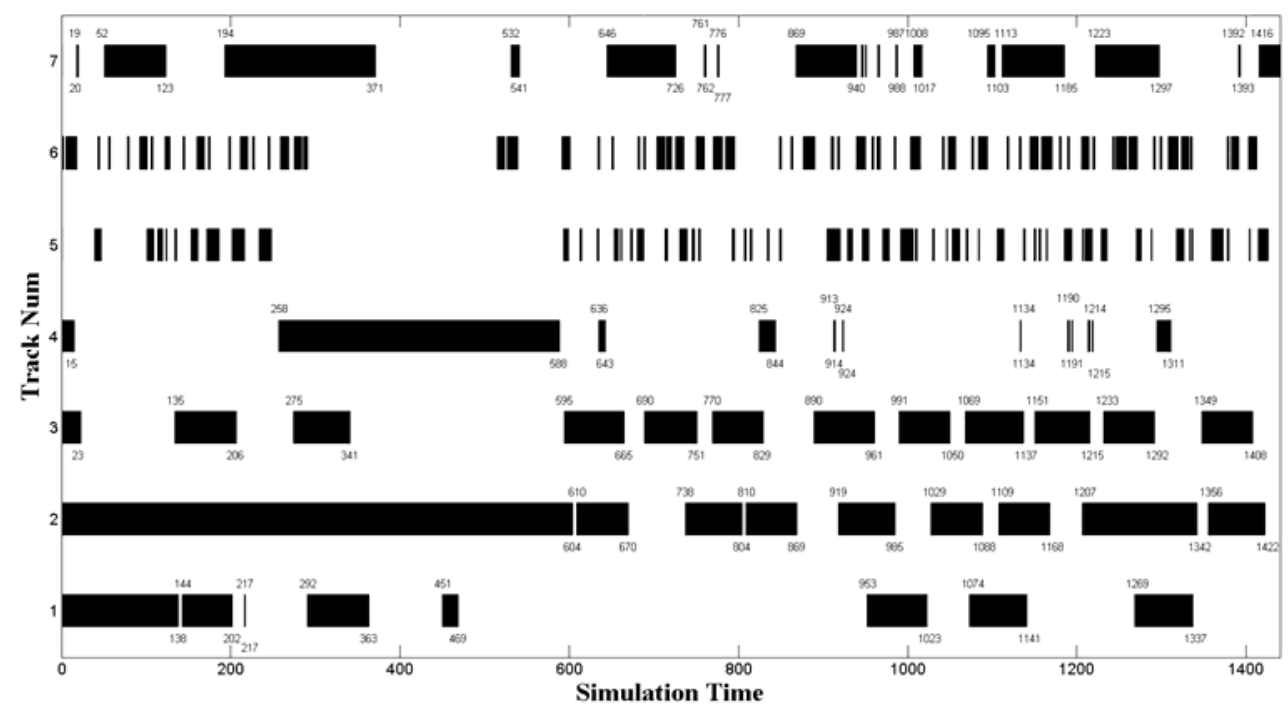

Figure 9. Scheme 8

Schemes 3, 10 to 13 study the effect of train combination operation on the capacity of the station. It can be seen that with the increase of the number of combined trains, the average occupancy time of the track, switch increased. Due to the limit of the length of the track, this article only set up 3G track to be the only track on witch can do combination operation, from the plan 13 (as shown in Figure 10) that the station in a day to handle the maximum number of train combination operation is 5 times. 


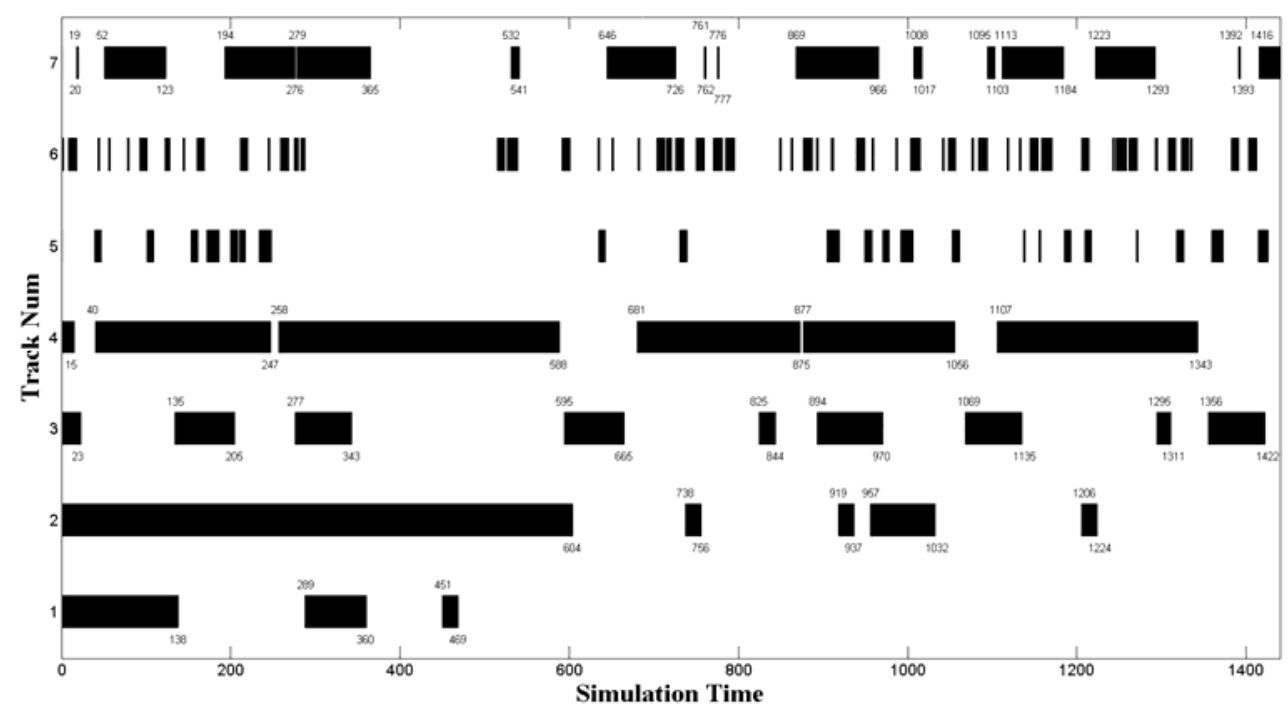

Figure 10. Scheme 13

\section{Conclusion}

In this paper, the station simulation system is established on the basis of the operation process of the station, based on the fixed and flexible application principle and the train insertion method. The Baoshen line CiyaoWan Station is used as an example to simulate the capacity of the station.

The simulation results show that the maximum capacity of the station and the maximum ability of the combined operation can be obtained by the simulation system evaluation and optimization. At the same time, the capacity of the station is strengthened by inserting the train to make use of the available time of the station's track and increasing the number of the route occupancy.

\section{References}

[1]. Chen Tao. Research on the theory and method of capacity calculation for high speed railway station [D]. Southwest Jiao Tong University, 2016.

[2]. Kang Liujiang. Optimization modeling and solving of the utilization of the throat area of the railway passenger station [D]. Beijing Jiaotong University, 2013.

[3]. Chen Xiaozhu. Simulation and analysis of the point line capacity coordination of high speed railway [D]. Southwest Jiao Tong University, 2013.

[4]. Wang Kebao. Line and the existing railway station connection with optimization study of [D]. of Southwest Jiao Tong University passenger transport, 2011.

[5]. Yang Yungui. Research on the calculation method of railway station capacity and investigation and calibration technology [D]. Southwest Jiao Tong University, 2010.

[6]. Li Dong. Research on the passing capacity of Heavy Haul Railway Technical Station [D]. Southwest Jiao Tong University, 2015.

[7]. Miao Jianrui, Jiang Xi, Yu, Zhao Yane, Yang Zhaoxia. Research on the simulation of the train arrival and shunting process of railway station (field) train [J]. 